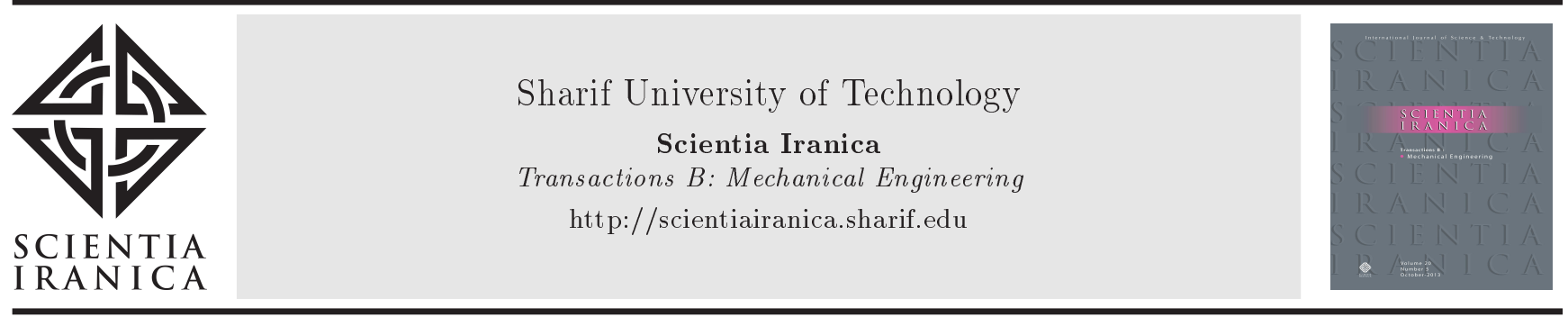

\title{
CFD investigation of an axial compressor with casing treatment for the enhancement of the stall margin
}

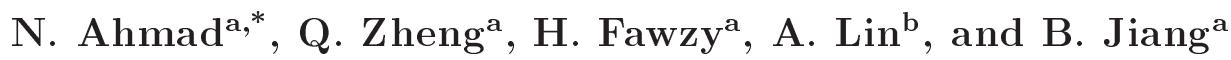 \\ a. College of Power and Energy Engineering, Harbin Engineering University, Harbin 150001, P.R. China. \\ b. School of Power and Energy, Northwestern Polytechnical University, Xi'an 710129, P.R. China. \\ Received 25 July 2020; received in revised form 8 November 2020; accepted 12 April 2021
}

\section{KEYWORDS \\ Axial compressor; Adiabatic efficiency; \\ CFD; \\ Casing treatment; \\ Stall margin.}

\begin{abstract}
Casing treatment is an efficient technique which is used to increase the compressor stall margin with a minor reduction in the efficiency. Various researches have indicated that the interaction between the blade passage and the groove is the main cause of the stall margin improvement. In this study a numerical study is conducted on a single circumferential casing groove using NASA rotor 37. The performance of the two circumferential groove models is studied by discretizing RANS 3D equations using a finite volume technique. The inception of the stall is predicted according to the criteria of convergence. Two models of the circumferential groove have been suggested and numerically tested. A single passage simulation is selected for the two models. The performance of the smooth casing and the two models are analyzed. Moreover, the stall margin, total pressure ratio and peak adiabatic efficiency of the normal casing, and the two models are analyzed to determine the influence of the groove on the axial compressor performance and stability. Models 1 and 2 stall margins are enhanced by $6.62 \%$ and $4.45 \%$ respectively. The adiabatic efficiency of Models 1 and 2 is decreased by $0.79 \%$ and $1.08 \%$ respectively.

(C) 2021 Sharif University of Technology. All rights reserved.
\end{abstract}

\section{Introduction}

The rudimentary factor of the axial compressor instability is the Tip Leakage Vortex (TLV) near to the shroud region. Several researchers have used experimental and numerical methods to divulge the mechanism of the stall and to extend the operating range of the axial compressor. The stall was evoked at the compressor blade Leadin Edge (LE) due to the TLV breakdown by Schlechtriem and Lötzerich [1]. The rotating instability mechanism of low-speed compressor was examined by Marz et al. [2] through experimental

*. Corresponding author. Tel.: +8613045175980 E-mail address: naseem_saddiqui@hrbeu.edu.cn (N. Ahmad).

doi: $10.24200 /$ sci.2021.56486.4742 and numerical techniques. The investigation demonstrated that the flow of the tip gap triggered the vortex structure near the compressor blade LE. This vortex formation caused unsteadiness that leads to rotating instability. With and without single circumferential groove, the stall inception features were experimentally investigated by Juan et el. [3] in a low-speed axial compressor. The stall inception of the spike type initiated for the both cases is a short scale disturbance.

Passive flow casing treatment is adopted to control the blade TLV for the improvement and stability of axial compressor. Various techniques have been proposed to enhance the axial compressor stall margin. Bailey [4] tested various circumferential grooves with a solid wall. Aluminium casing inserts were used for the modification. The different number of grooves, depth, and locations were changed and the results indicated that stall margin and stability were changed 
with different modifications. Heinichen et al. [5] used only one groove for three different compressor rotors.

The results revealed that the working range had been increased for all three compressor rotors and the stall occurred due to shock vortex interaction. The influence of a single groove casing was investigated by Shi et al. [6]. The results explained that a single groove plays an important role close to the LE of the rotor blade; the groove stabilized the flow close to stall limit and increased the stall margin of the compressor. Li et al. [7] carried out experimental work with a single groove to investigate the unsteady pressure in the blade tip region. They reported that unsteady pressure and TLV were weakened with the installation of the circumferential groove, that helped to improve the stall margin.

The influence of a single groove for two different compressors was investigated by Yasunori et al. [8]. The stall and flow fields were analyzed for two different compressors. The results showed that the blockage flow was different for the two compressors. Coupling technique based on casing treatments was used by Wang et al. [9] to improve the compressor performance. They reported that Coupling Casing Technique (CCT) enhanced $16.7 \%$ compressor stability. $\mathrm{Xi}$ et al. [10] suggested new approach for the casing treatment of compressor stability. A parametric study was conducted by Dinh et al. [11] to connect the circumferential grooves with the blade tip injection. The groove height, front length and rear length were selected as geometrical parameters. They reported that stable range extension and stall margin have less effect on the ejection mass flow rate than the geometrical parameters, whereas the injection with circumferential grooves significantly enhanced the efficiency and stall margin.

Azizi et al. [12] developed an algorithm for the aerodynamic design of the axial compressor. The algorithm was used with the help of FORTRAN programming and evaluated the results of NACA eight stage compressor. Slot based casing treatment was adopted by Zhang et al. [13] to enhance subsonic axial compressor stability. The results revealed that the stall margin improvement of the experiment and simulations increased by nine and eight percent respectively whereas the maximum efficiency drop by eleven percent for both experiment and simulations. A special algorithm and experimental procedure were used by Le et al. [14]. They recorded the interaction of groove passage with the help of velocity component and frequency spectrum. Li et al. [15] studied experimentally inlet radial distortion using three stage axial compressor to improve the aerodynamic stability. They concluded that inlet radial distortion influenced the first stage flow field, enfeebled at the second stage, and totally unaffected at the third stage of the axial compressor.
A new Ball Spine (BS) algorithm was developed by Madadi et al. [16] for axial compressor blade passage. Double Circular Arc (DCA) blade profile has been tested with the suggested BS algorithm and the gained geometry is in line with the target geometry.

Wang et al. [17] worked on recirculating casing treatment experimentally and numerically using low speed axial compressor. They reported that recirculating treatment increased the stall margin and performance of the compressor. Axial compressor was optimized with casing grooves by Kim et al. [18]. The results demonstrated that the optimum design increased the stall margin by $5.13 \%$ whereas the adiabatic efficiency was decreased by $0.18 \%$.

The self-circulating casing treatment that revealed the injector position was investigated by Yan and Wuli [19]. They reported that TLV delayed the deterioration of the tip flow field and increased rotor performance. Kawase and Aldo [20] used recirculation channel to enhance the stall margin of axial compressor. The results demonstrated that $18.2 \%$ axial position of fluid improved the stall margin by $5.5 \%$. Multi-objective optimization was carried out on NASA rotor 37 by Zhao et al. [21]. This technique increased the stall margin of the compressor. Moreover, the TLV strength and Boundary Layer Separation (BLS) could be decreased with this optimization techniques.

A hybrid slot-groove in the axial compressor was experimentally investigated by $\mathrm{Li}$ et al. [22]. The results revealed that slots-grooves hybrid CT could enhance the stall margin of the compressor by $19.79 \%$ and the efficiency decreased by $1.5 \%$. A single groove was used by Mao and Liu [23] to improve the counter rotating axial compressor performance. They reported that a single groove reduced the TLF structure and improved stability. Casing grooves with various tip clearances gap were investigated by Naseem et al. [24] and it was found that the performance of small tip clearance is better than large tip clearance. Zhu and Yang [25] adapted multi-objective optimization to coupled slot and grooves to improve the compressor stability. The result showed that compressor stability can be increased with slot-groove casing treatment. Du et al. [26] investigated experimentally and numerically a single groove casing treatment at various locations to increase the axial compressor performance and stability. The results elucidated that the ideal location of the blade chord is $56 \%$ for the stall margin enhancement.

Liu et al. [27] used an algorithm to obtain the velocity average magnitude using hot-wire probes. The experimental data revealed that the interface of the blade tip flow with circumferential grooves is the key for the stability and performance compressor. Recently Naseem et al. [28] worked on NASA rotor 37 to increase the axial compressor stall margin. Their results demonstrated that the stall margin is enhanced 
with the casing treatment. The influence of different trench design on axial compressor is investigated by Ashok et al. [29]. They reported that the introduction of different trenches increased the compressor stability. Wang et al. [30] proposed a three-step method to find the location of stall limiting to understand the stall mechanism. Their results revealed that couple casing treatment is very effective to improve the compressor stability. Hamzezade et al. [31] used multistage compressor with different tip gaps for casing treatment. They concluded that the maximum stall margin is achieved with a minimum tip gap size. However, the previous researchers used a single groove with different axial locations instead of using a single groove with fixed location.

The main objective of the present research is to investigate numerically the impact of a single circumferential groove with a fixed position by deploying the finite volume technique with the help of RANS 3D equations. Two models have been tested numerically. The stall margin, adiabatic efficiency and total pressure ratio of the two models are analyzed to assess the influence of the single circumferential groove on the axial compressor stability and performance.

\section{Design conditions of the tested compressor rotor}

NASA axial transonic rotor 37 [32] is chosen for the present research study. At NASA Lewis Research Center, an inlet rotor 37 was designed for the transonic axial compressor in the late 1970s. The measurement of the flow was performed for a single stage of the rotor and at the ASME conference the results of the CFD code assessment were provided for the blind test case. The compressor rotor design conditions are given in Table 1. The compressor rotor consists of 36 blades having the pressure ratio 2.106 . The rotor polytropic

Table 1. Test compressor design specifications [32].

\begin{tabular}{lc}
\hline Design specification & Value \\
\hline Blades number & 36 \\
Total pressure rise ratio & 2.106 \\
Solidity at tip & 1.29 \\
Mass flow (design) (kg/s) & 20.19 \\
Inlet hub-to-tip diameter ratio & 0.7 \\
Rotational speed of rotor (rpm) & 17188.7 \\
Choked mass flow (kg/s) & 20.93 \\
Mach number at inlet hub & 1.13 \\
Reference temperature (K) & 288.15 \\
Tip clearance (mm) & 0.356 \\
Tip speed (m/s) & 454.136 \\
Rotor polytropic efficiency & 0.889 \\
\hline
\end{tabular}

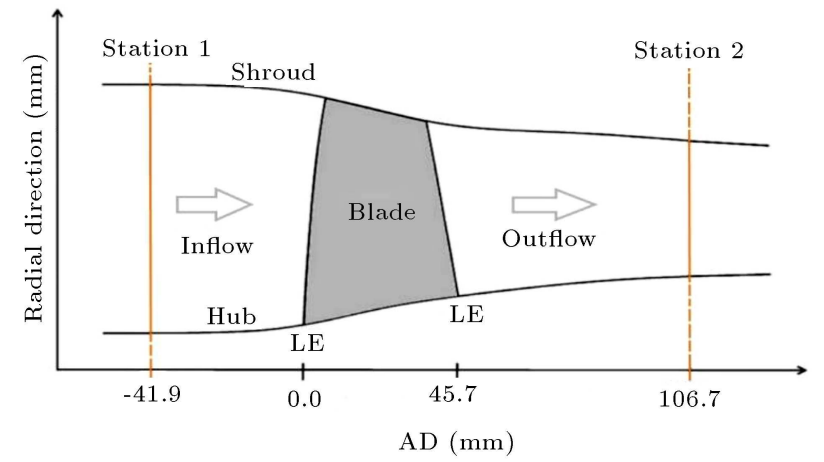

Figure 1. NASA rotor 37 meridional view.

efficiency is 0.889 with the rotational speed of 17188.7 $\mathrm{rpm}$. The mass flowrate at the design is $20.19 \mathrm{~kg} / \mathrm{s}$ and $20.93 \mathrm{~kg} / \mathrm{s}$ is the choked mass flowrate. The tip clearance gap at forty-seven span is $0.356 \mathrm{~mm}$. The compressor rotor meridional view is illustrated in Figure 1. The parameter like total temperature and total pressure at the inlet (Station 1) is measured that is related to the mass flowrate at the outlet (Station 2). The distance between the upstream Station 1 and LE of the blade is $-41.9 \mathrm{~mm}$ and at the downstream close to the hub Station 2 is located with a distance of $106.7 \mathrm{~mm}$ from the Trailing Edge (TE) of the compressor blade.

\section{Design and explanation of the Circumferential Groove Casing Treatment (CGCT)}

Two models of a single groove are designed and tested numerically for rotor 37 in the present research study to improve the performance and stable range and it is represented in Figure 2. The performance and stable operating range of Models 1 and 2 are compared with smooth wall casing and each other. Both models consist of a single rectangular groove and the groove is located from 10 to $90 \%$ of axial chord. The groove height of Model 1 is $1 \mathrm{~mm}$ and it covers the area of $80 \% c_{x, r}$, whereas the groove height of Model 2 is $1.5 \mathrm{~mm}$ and it also covers the area $80 \% c_{x, r}$. Former researchers Kim et al. [33] and Huang et al. [34] worked on the depth and numbers of circumferential grooves using several grooves to enhance the axial compressor stall margin. Their research work revealed that CGCT

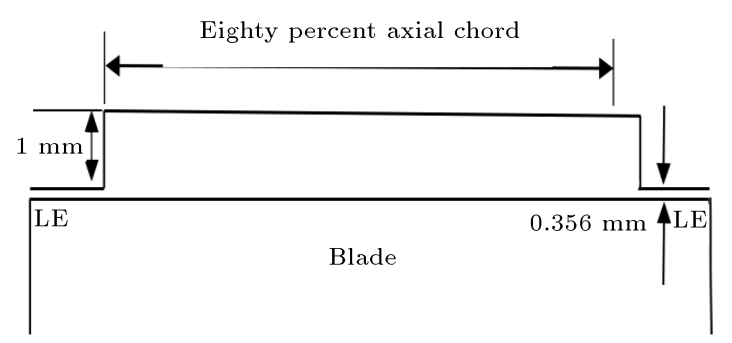

Figure 2. The circumferential groove axial location. 
could increase the axial compressor stall margin, stability and performance. The rudimentary model of the present research work is Model 1 that explores the influence of a single groove in axial compressor performance.

\section{Governing equations and numerical method}

The $k-\varepsilon$ turbulence model is used in numerical simulations. The $k-\varepsilon$ model presents two new variables into the system of equations. The continuity equation is written as:

$$
\frac{\partial \rho}{\partial t}+\frac{\partial}{\partial x_{i}}\left(\rho U_{i}\right)=0
$$

The equation of momentum becomes:

$$
\begin{array}{r}
\frac{\partial}{\partial t}\left(\rho U_{i}\right)+\frac{\partial}{\partial x_{j}}\left(\rho U_{i} U_{j}\right)=-\frac{\partial p}{\partial x_{i}}+\frac{\partial}{\partial x_{j}} \\
{\left[\mu_{e f f}\left(\frac{\partial U_{i}}{\partial x_{j}}+\frac{\partial U_{j}}{\partial x_{i}}\right)\right]+S_{M},}
\end{array}
$$

where $\mu_{e f f}$ is the effective viscosity accounting for turbulence, $S_{M}$ is the sum of the body forces and $p^{\prime}$ is the modified pressure and it is defined as follow:

$$
p^{\prime}=p+\frac{2}{3} \rho k+\frac{2}{3} \mu_{e f f} .
$$

The $k-\varepsilon$ model is based on the concept of the eddy viscosity, therefore:

$$
\mu_{e f f}=\mu_{+} \mu_{t},
$$

where $\mu_{t}$ is the turbulence viscosity. In the $k-\varepsilon$ model it is considered that the turbulence viscosity is related to the turbulence kinetic energy, dissipation, and the relation as:

$$
\mu_{t}=C_{\mu} \rho \frac{k^{2}}{\varepsilon}, \quad C_{\mu}=0.09
$$

The values of $k$ and $\varepsilon$ are derived straightly from the differential transport equations for the turbulence kinetic energy and turbulence dissipation rate, which are obtained as follows:

$$
\begin{gathered}
\frac{\partial(\rho k)}{\partial t}+\frac{\partial}{\partial x_{j}}\left(\rho k U_{j}\right) \\
=\frac{\partial}{\partial x_{j}}\left[\left(\mu+\frac{\mu_{t}}{\sigma_{k}}\right) \frac{\partial k}{\partial x_{j}}\right]+P_{k}-\rho \varepsilon+P_{k b}, \\
\frac{\partial(\rho \varepsilon)}{\partial t}+\frac{\partial}{\partial x_{j}}\left(\rho \varepsilon U_{j}\right)=\frac{\partial}{\partial x_{j}}\left[\left(\mu+\frac{\mu_{t}}{\sigma_{\varepsilon}}\right) \frac{\partial \varepsilon}{\partial x_{j}}\right] \\
+\frac{\varepsilon}{k}\left(C_{\varepsilon 1} P_{k}-C_{\varepsilon 2} \rho \varepsilon+C_{\varepsilon 1} P_{\varepsilon b} .\right.
\end{gathered}
$$

$P_{k b}$ and $P_{\varepsilon b}$ denote the effect of the buoyancy forces.
$P_{k}$ is the production turbulence due to viscous forces. The commercial solver flow Ansys 17 [35] is used for all the numerical simulations. With the help of the finite volume method, the equations of 3D RANS are discretized in the computational domain. The equations are tested in a rotating frame of reference. Multiblock structure grids are generated by AutoGrid5 NUMECA [36]. Ansys CFX is used for numerical solution and post-process analysis. Ansys design modeler is used for the fluid domain and circumferential casing groove, and Ansys ICEM CFD is used for the structured mesh, respectively. One passage computational domain is adopted for the present research work by imposing periodic conditions on the circumferential direction boundaries. One passage multiblock structured grids are created. The structure of the computational grids is illustrated in Figure 3. For structure blocks, H-O$\mathrm{H}$ topology is adopted. $\mathrm{O}$ type structure grids are created at the compressor blade LE and TE. The main flow region is constructed with $\mathrm{H}$ type of grids. The minimum grid spacing is set to $3.0 \times 10^{-6} \mathrm{~m}$ to captured $y+<10$ at the surface of solid walls. The number of structure grids of both the inlet block and outlet block is $21 \times 29 \times 40$. Furthermore, the tip clearance gap is discretized with eighteen grid cells in the radial direction with a constant radial distance. The total number of nodes in the present research study is $4.8 \times 10^{5}$ and $30 \times 29 \times 70$ number of structure grids are used for single-groove block. To eradicate the influence of the grid numbers on the compressor rotor flow field, a grid sensitivity test is performed for rotor 37. Total pressure, $101325 \mathrm{~Pa}$, and total temperature,

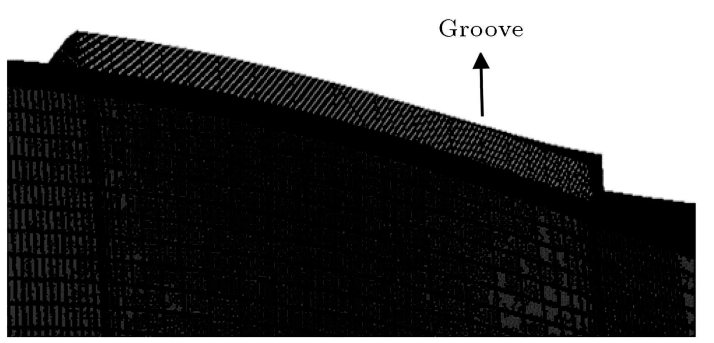

(a) Single circumferential groove

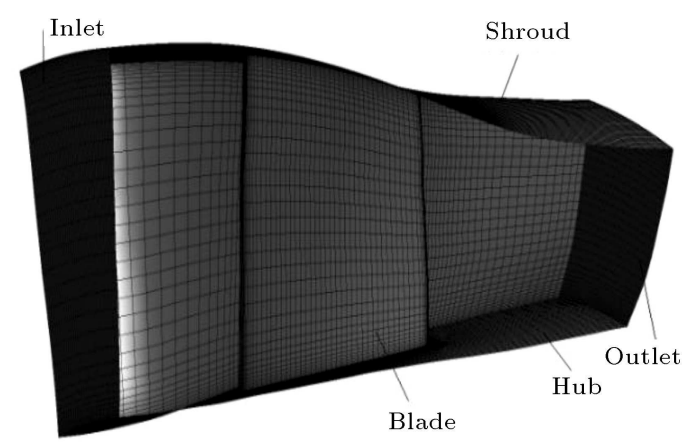

(b)

Figure 3. The computational mesh of rotor 37 . 


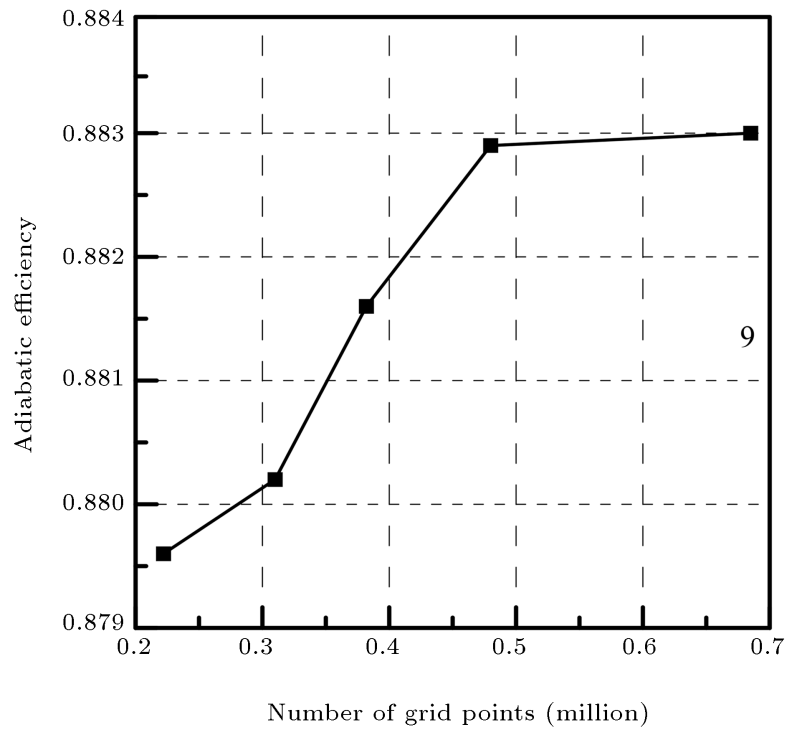

Figure 4. Simulation results with a different number of grids.

$288.15 \mathrm{~K}$, are given in the rotor inlet boundary details. Static pressure is changed at the rotor outlet boundary details. Five different cases of the grid numbers are simulated for sensitivity. The calculated rotor adiabatic efficiency is depicted in Figure 4. For adiabatic efficiency, there is slight change from 0.48 million to 0.685 million. Moreover, the grids sensitivity of static pressure contours at $98 \%$ span for five cases (near stall limit) is depicted in Figure 5. In Figure 5(d) and (e) it can be observed that the static pressure is slightly changed from 0.48 million to 0.685 million grids. Given the computational cost and accuracy, grid points of 0.48 million are selected for this research work. $k$ epsilon turbulence model is adapted for all the numerical simulations. At the inlet of the compressor the total temperature, flow angle and total pressure are specified with the intensity of turbulence of $5 \%$. The boundary conditions for the rotor blade are selected as smooth and adiabatic with no-slip wall. The radial equilibrium state is fixed at the outlet. At the outlet of the compressor rotor, backpressure is increased by setting the condition of the state of radial equilibrium to analyze radial distribution of the static pressure. The point near to the stall condition is considered the final steady point. The single circumferential groove and the rotor blade passage are connected with the method of GGI (General Grid Interface).

\section{Results and discussion}

The adiabatic efficiency and total pressure ratio validation are illustrated in Figure 6. The validation for the adiabatic efficiency and total pressure ratio are taken from the author's previous research [37]. The method of the casing groove in axial compressor is adopted for

stability and performance enhancement. To evaluate the axial compressor stability and performance, the adiabatic efficiency, stall margin enhancement, stall margin and total pressure ratio parameters are used in the present research study. These parameters can be explained as follows:

$$
P R=\frac{P_{t, \text { out }}}{P_{t, \text { in }}}
$$

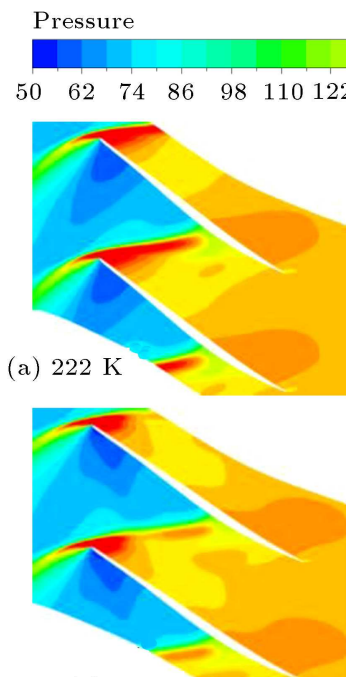

(c) $380 \mathrm{~K}$

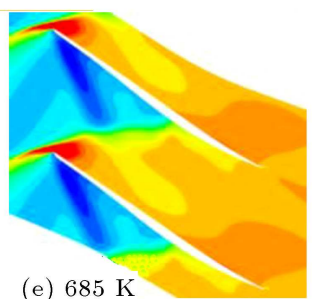

(e) $685 \mathrm{~K}$

Figure 5. Grids sensitivity of static pressure contours at $98 \%$ span for five cases (near stall limit).

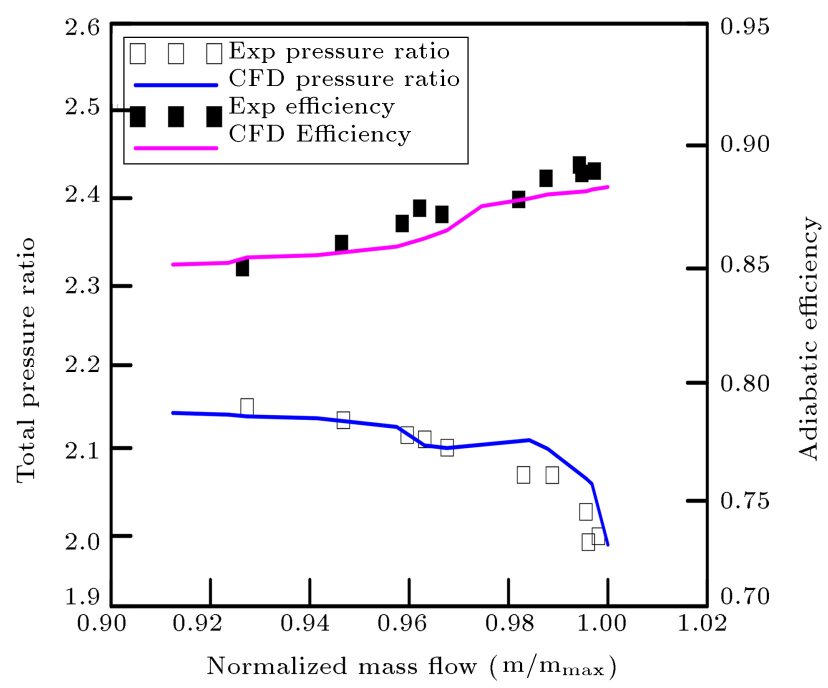

Figure 6. Compressor map validation of NASA rotor 37 [37]. 


$$
\begin{aligned}
& \eta_{\text {peak }}=\left(\frac{\left(\frac{P_{\text {total }, \text { out }}}{P_{\text {total } \text {,in }}}\right)^{\frac{\gamma-1}{\gamma}}}{\left(\frac{T_{\text {total,out }}}{T_{\text {total }, \text { in }}}\right)}-1\right), \\
& S M=\left(\frac{\dot{m}_{\text {peak }}}{\dot{m}_{\text {stall }}} \times \frac{P R_{\text {stall }}}{P R_{\text {peak }}}-1\right) \times 100 \%, \\
& S M I=S M_{C G}-S M_{S W} .
\end{aligned}
$$

The difference between operating RPM and the revolution per minute that the blades of compressor rotor at altitude would stall and transitory acceleration is known as stall margin. Whereas $P R$ denotes the pressure ratio of the compressor and $\dot{m}$ represents the mass flowrate. The stall and peak subscripts in Eq. (10) signify the total pressure ratio and mass flowrate at peak and near stall conditions correspondingly. $\mathrm{Tt}, \mathrm{Pt}$ and $\lambda$, represent total temperature ratio, total pressure ratio and specific heat, respectively.

Due to low mass flowrate, tip leakage flow and TLV go to stagnation zone produced passage shock in downstream region that caused vortex breakdown. The extreme blockage has occurred in the area of main flow region results in instability in the axial compressor. The TLV breakdown in NASA rotor 37 is a general phenomenon that was reported by many researchers $[11,33,34]$. Model 1 is selected as the fundamental model for the present research work to determine the influence of a single groove on the axial compressor performance and stability.

The parameters for the performance of the transonic compressor are efficiency, stall margin improvement, stall margin and total pressure ratio. The near stall limit of Model 1 and the smooth casing is 0.898 and 0.912 (normalized mass flowrate), respectively. The total pressure ratio of Model 1 and smooth casing is 2.15 and 2.14 close to the stall condition, which is in line with the normal casing.

The radial performance distribution of adiabatic efficiency at $98 \%$ choked flow is shown in Figure 7 . The adiabatic efficiency performance is compared with the experimental data for smooth casing and Model 1. It can be observed in Figure 7 that adiabatic efficiency of the smooth casing and Model 1 is lower from the blade hub to $30 \%$ span but in the middle, this match well the experimental data. So, the simulation results of smooth casing and Model 1 are in good agreement with the experimental data. Radial distribution of circumferential area averaged flow angle at $98 \%$ choked flow is shown in Figure 8. It can be seen in Figure 8 that the flow angle for smooth casing and Model 1 is lower at the hub. After $10 \%$ span the flow angle for smooth casing and Model 1 shows similar behaviour to the experimental data. Overall, the flow angle calculated through simulations for smooth casing and Model 1 are in line with experimental data.

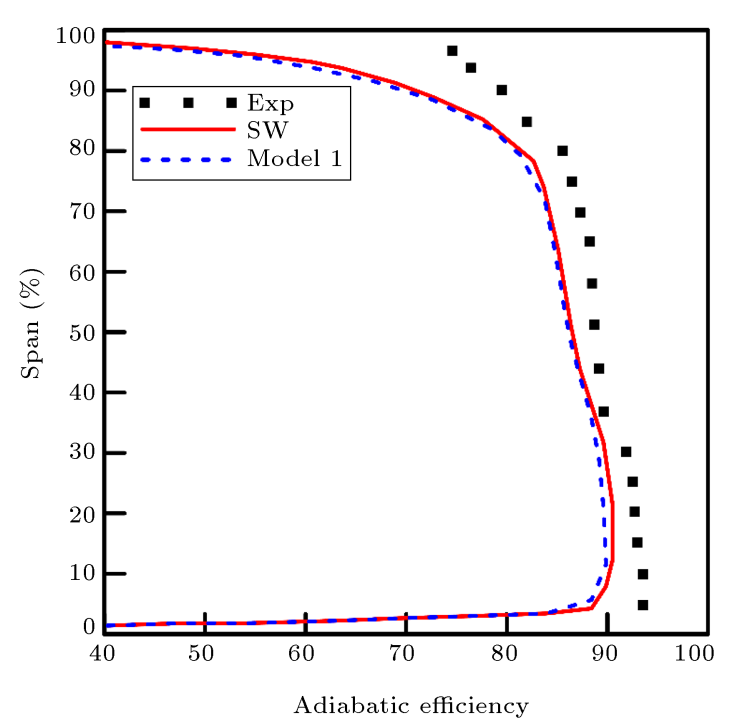

Figure 7. Radial performance distribution of adiabatic efficiency at $98 \%$ choked flow.

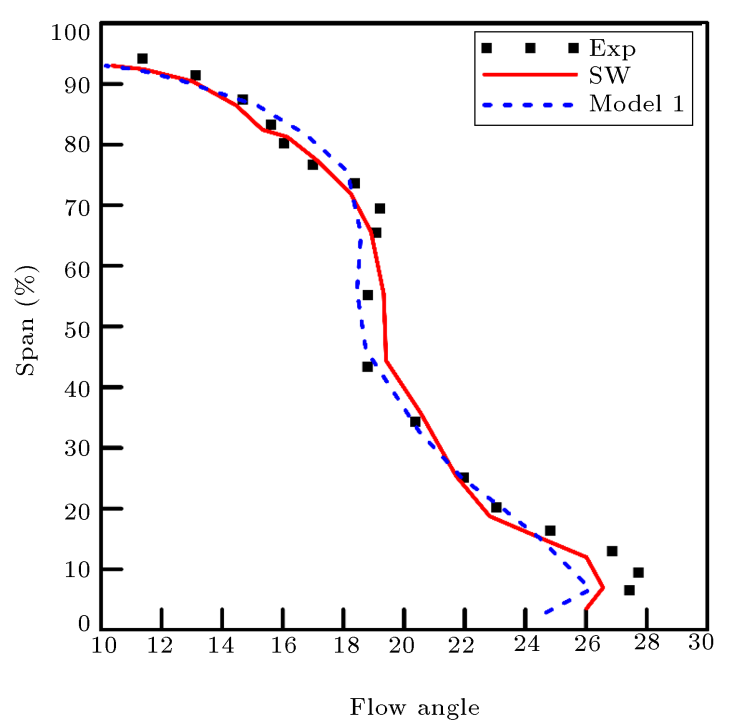

Figure 8. Radial distribution of circumferential area averaged flow angle.

The Mach number contours of Models 1 and 2 and smooth casing close to the stall limit are shown in Figure 9. The circumferential groove has also influenced on the momentum transport through the compressor blade tip. The separation occurred at the Suction Side (SS) of the compressor rotor blade with the normal casing as depicted in Figure 9(a). With the help of the circumferential groove, the compressor blade suppressed the separation in Figure 9(b) and (c), respectively. The Mach number for Models 1 and 2 is recuperated at the compressor rotor blade suction side. High momentum flux is formed with the installation of the circumferential groove from the Pressure Side (PS) of the compressor rotor blade to SS. So, the separation is suppressed on the compressor blade SS due to the 


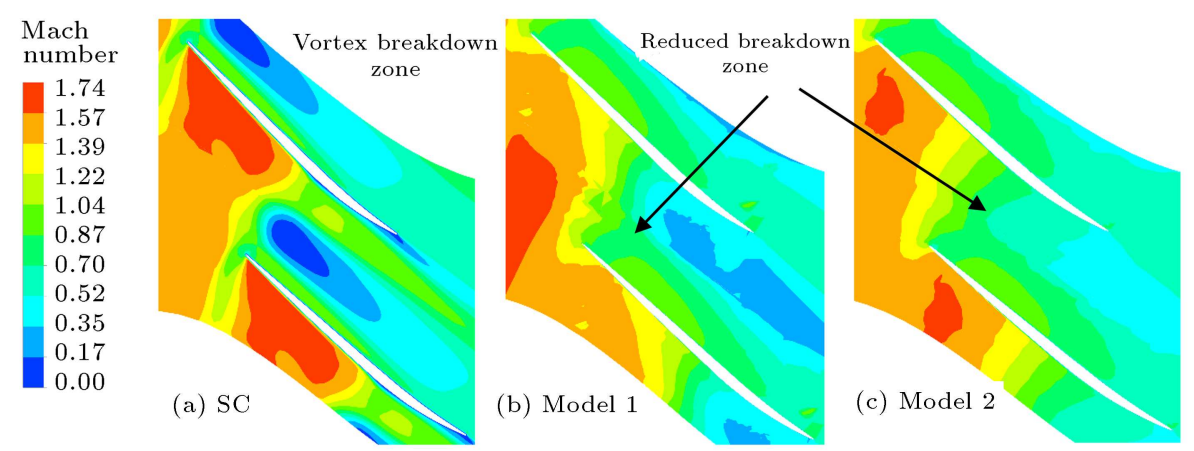

Figure 9. Mach number contours: (a) Smooth Casing, (b) Model 1, and (c) Model 2 (near stall point at 98\% span).

installation of the circumferential groove and enhance the axial compressor stability.

The compressor blade loading distribution of Model 1 and smooth wall casing close to the stall limit is shown in Figure 10. The variance of pressure is observed in the groove installation region between the PS and SS of the compressor rotor. There are two important effects of the installed circumferential groove. First of all, the pressure oscillation on the PS that caused to rise the local pressure similar to a wave pattern. The second effect which is the downward movement of the shockwave, seen from the pressure distribution on the SS of the compressor blade, gives benefit to the compressor stability. It indicates that fixing the groove increase the compressor loading and shockwave leads to the rotor blade passage which cause to increase the blade pressure ratio and the flux ratio decreased at the stall point. Near the LE of blade, the flow angle of the tip clearance is changed for smooth casing and Model 1, whereas the rear part shows a similar shape because at the rear parts no groove

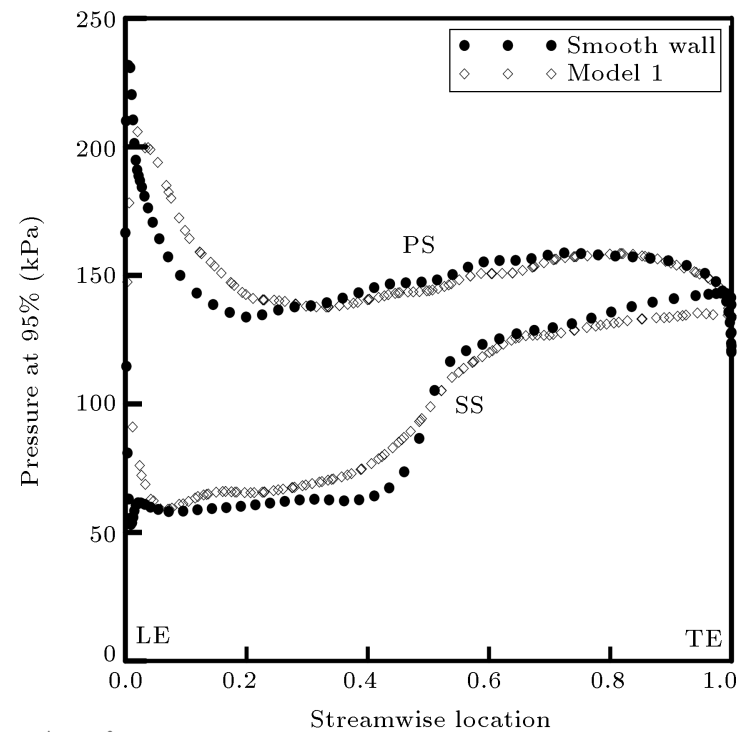

Figure 10. Streamwise distribution of pressure for smooth wall casing and Model 1 at $95 \%$ span (near stall limit). is installed. More pressure variation at the groove location can be seen in Model 1 shown in Figure 10.

It can be seen in Figure 11 that the installation of the casing groove minimized the blockage. The streamlines for the smooth wall casing and Models 1 and 2 are shown in Figure 11. The separation can be observed in Figure 11 close to the LE of the compressor blade PS. The spillage phenomenon at the blade LE occurred due to separation. Near the LE of the blade pressure side, the TLF caused the low fluid zone (LE blockage) which is linked to rotating stall. With the help of the circumferential groove, the separation is suppressed as depicted in Figure 11(b) and (c). The PS near the blade LE suppressed the separation with the help of circumferential groove that can be seen in Figure 11(b) and (c). High momentum flux is produced on the blade PS to SS. This helped in enhancement of the compressor stability and performance.

The pressure ratio of Models 1 and 2 and smooth wall casing is $2.15,2.11$, and 2.14 , respectively. The pressure ratio of Model 1 is slightly greater than the smooth wall, whereas the pressure ratio of Model 2 is less than the smooth casing. Both pressure ratio agrees well with the normal casing. The stall margins of normal casing and Models 1 and 2 are illustrated in Figure 12. The stall margins of Models 1 and 2 are $19.89 \%$ and $17.72 \%$, respectively. Models 1 and 2 stall margins are increased by $6.62 \%$ and $4.45 \%$, respectively. The stall margin achieved in the present study is higher than the stall margin reported by $[11,33]$.

The bar chart of the adiabatic efficiency for Models 1 and 2 and smooth wall casing is shown in Figure 13. The adiabatic efficiencies of Models 1 and 2 , and smooth wall casing are $87.52 \%, 87.23 \%$, and $88.31 \%$, respectively. It can be seen in Figure 13 that the adiabatic efficiency is slightly reduced with the installation of a single groove. The efficiencies of Models 1 and 2 are reduced by $0.79 \%$ and $1.08 \%$, respectively. The adiabatic efficiencies achieved by Model land 2 are higher than the adiabatic efficiencies investigated by $[33,11]$.

Contours of entropy at $97 \%$ span for Models 1 

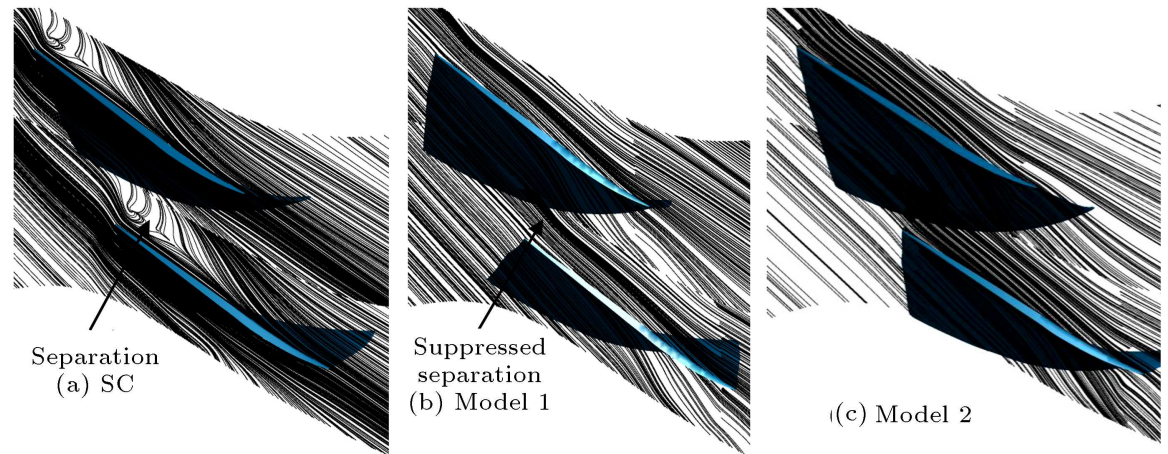

Figure 11. Streamlines contours at 98\% (span): (a) Smooth casing, (b) Model 1, and (c) Model 2 (near stall point).

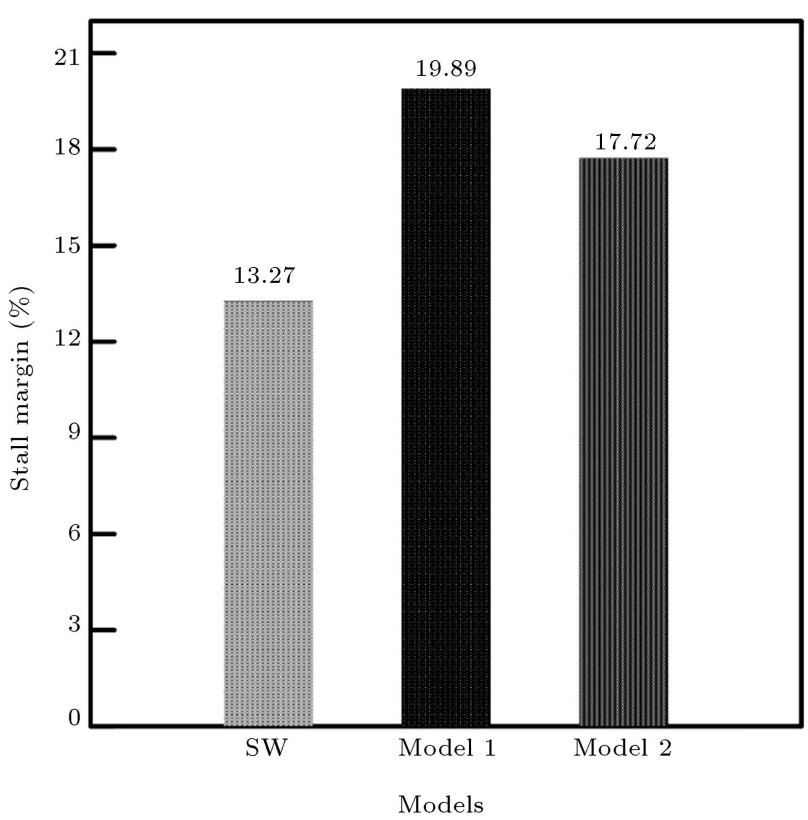

Figure 12. A comparative bar chart of stall margin for the smooth casing and Models 1 and 2.

and 2, and smooth casing are shown in Figure 11 (near stall limit). Figure 14 shows a high value of entropy close to the blade tip for Models 1 and 2. With fixing the circumferential groove, Models 1 and 2 show lower value of entropy generation in the downstream region, and the smooth casing does not exhibit this behavior. The single circumferential groove is installed on Models 1 and 2 from 10 to $90 \%$ blade chord. The height of the groove of Models 1 and 2 is 1 and $1.5 \mathrm{~mm}$, respectively. The simulation results are in good agreement with [32]. The entropy reduction for Models 1 and 2 indicates the improvement of the TLV structure. The adiabatic efficiency is recovered in low mass flow region for Models 1 and 2 due to the installation of the circumferential groove. Figure 14(b) indicates the lower value of the entropy in the downstream region than that in the smooth casing and Model 2. So, the TLV structure and reduction of entropy generation is more enhanced in Model 1, compared to smooth casing

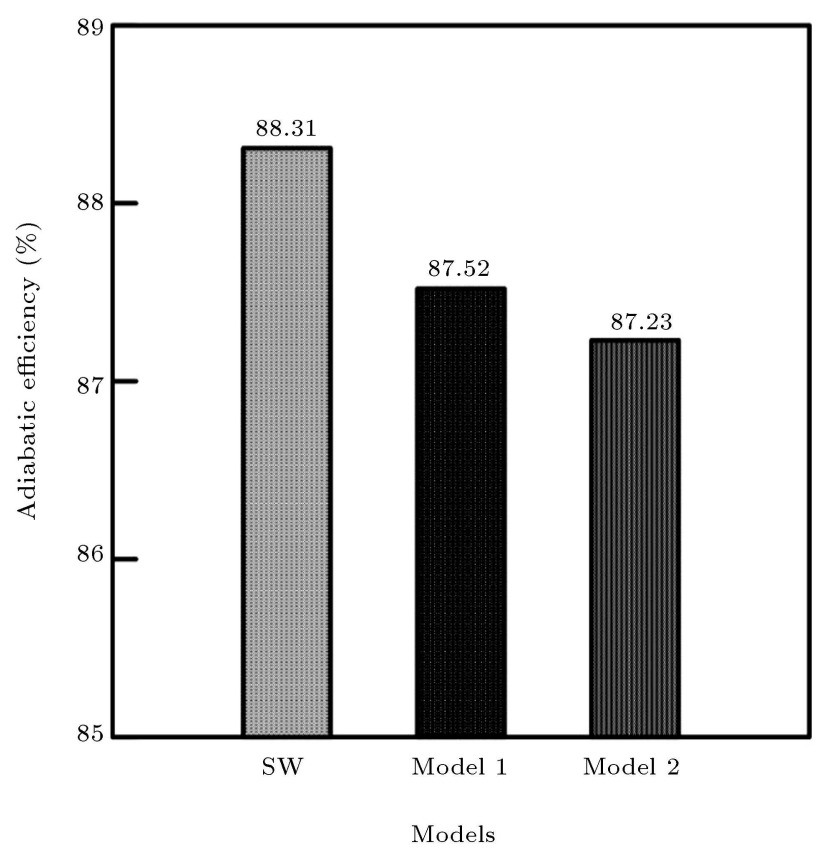

Figure 13. A comparative bar chart of adiabatic efficiency of the smooth casing and Models 1 and 2 .

and Model 2. The reduction of entropy generation and TLV structure are also improved for Model 2 due to the installation of the circumferential groove but with less efficient in comparison to Model 1. Comparing smooth casing and Models 1 and 2, Model 1 is a more effective model with reduced TLV structure. Furthermore, stall margin of Model 1 is much higher than that of the normal casing and Model 2 because of the improved TLV structures in low mass flow region with additional efficiency recuperation.

Contours of the axial velocity near stall point at $98 \%$ span, for the smooth wall casing and Models 1 and 2, are illustrated in Figure 15. In the axial compressor, the intensity interaction between the shock wave and the TLV is increased when the mass flow rate is decreased and this goes to downstream in the low-speed region. Therefore, the loss flow energy has occurred in the blade passage shock. The area of low-speed 

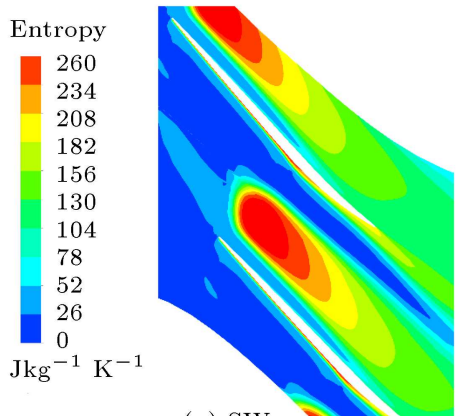

(a) SW
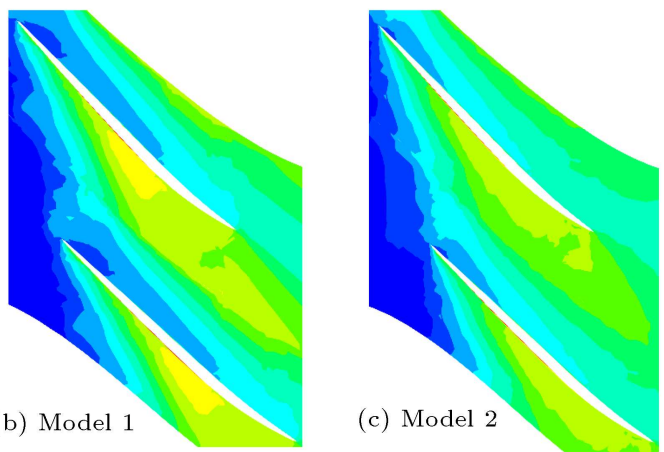

(c) Model 2

Figure 14. Contours of entropy: (a) Smooth casing, (b) Model 1, and (c) Model 2 (near stall point at 98\% span).
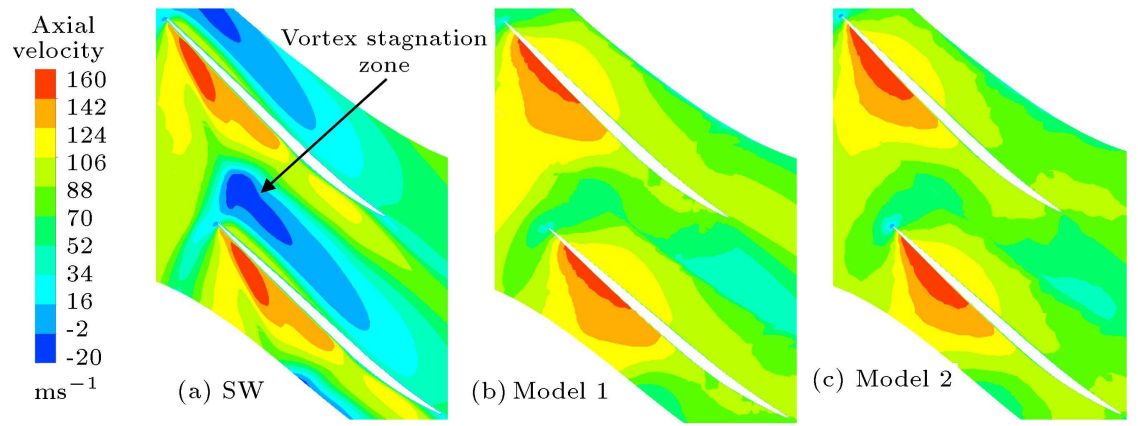

Figure 15. Contours of axial velocity at (a) Smooth casing, (b) Model 1, and (c) Model 2 (near stall point at $98 \%$ span).

region is known as vortex stagnation zone. The track of the main flow is changed with the vortex stagnation zone and the back pressure is increased which cause separation in the flow field and reduce the flow velocity. The vortex stagnation can be reduced with the help of CGCT. This indicates that circumferential grooves are useful to optimize the axial compressor performance and stability. The installation of the CGCT gives the alleviation of the vortex stagnation zone as seen in Figure 15(b). As depicted in Figure 15(c), the area of the blockage zone decreased in Model 2, enhance the compressor performance and stability. The single circumferential groove is installed on Models 1 and 2 from 10 to $90 \%$ of the blade chord. The heights of the grooves of Models 1 and 2 are $1 \mathrm{~mm}$ and $1.5 \mathrm{~mm}$, respectively. The value of the axial velocity at the tip of the rotor blade is higher for smooth wall casing whereas a similar pattern is not observed in Models 1 and 2 showing the enhancement and stability of axial compressor. Figure 15(b) indicates that among all the models, Model 1 is more effective due to higher stall margin and stability improvement.

\section{Conclusion}

The present research study numerically investigated the influence of a single groove on the total pressure ratio, stall margin and adiabatic efficiency using 3D RANS equations. Below, significant and important results are drawn from the present research study:
- The vortex stagnation zone is produced due to the intensity interaction between the shockwave and Tip Leakage Vortex (TLV), that had caused instability in an axial compressor. The stall greatly impacted on vortex stagnation zone;

- The separation of the Pressure Side (PS) from the blade LE substantially influences on the occurrence of the rotating stall;

- The installation of a circumferential groove minimizes the area of the vortex stagnation zone. The blade tip leakage flow is decreased with the installation of the circumferential groove;

- The circumferential groove minimizes the area of the vortex stagnation zone and this is advantageous to increase the stable range and performance of the compressor;

- It is observed in the contours of the numerical simulations that a single circumferential groove used in both models increases the axial compressor stall margin. With the increase of height of the circumferential groove, the axial compressor stable range and performance are changed. Increasing the height of the circumferential groove deteriorates the axial compressor performance and stability;

- In Model 1, the maximum stall margin is $19.89 \%$ and adiabatic efficiency is $87.52 \%$. Therefore, the performance of Model 1 is better than Model 2 and smooth casing; 
- The maximum adiabatic efficiency is slightly reduced for both models with the installation of the circumferential groove. Entropy is generated with the installation of CGCT which mixed with corner separation and reverses tip clearance flow caused a reduction in the adiabatic efficiency of the axial compressor;

- A single circumferential groove is installed covering the area from 10 to $90 \%$ of the blade chord. A total of $80 \%$ blade chord area is covered by the single groove. The blockage zone is effectively reduced with the help of a single circumferential groove in the mentioned location.

Some further investigations are needed to be done in the future regarding the optimization of the height of the circumferential groove.

\section{Nomenclature}

$\begin{array}{ll}\text { CFD } & \text { Computational Fluid Dynamics } \\ \text { PR } & \text { Total Pressure Ratio } \\ \text { SS } & \text { Suction Side } \\ \text { PS } & \text { Pressure side } \\ \text { EXP } & \text { Experiment } \\ \dot{m} & \text { Mass flowrate } \\ \text { RANS } & \text { Reynolds Averaged Navier Stokes } \\ \text { LE } & \text { Leading Edge } \\ \text { CGCT } & \text { Circumferential Grooves Casing } \\ & \text { Treatment } \\ \text { TE } & \text { Trailing Edge } \\ T_{t} & \text { Total temperature } \\ P_{t} & \text { Total Pressure } \\ \text { TLV } & \text { Tip Leakage Vortex } \\ \text { TCF } & \text { Tip Clearance Flow } \\ \text { TLF } & \text { Tip Leakage Flow } \\ C_{x, r} & \text { Blade tip axial chord } \\ \text { SMI } & \text { Stall Margin Improvement } \\ \text { BLS } & \text { Boundary Layer Separation } \\ \text { DCA } & \text { Double Circular Arc } \\ \gamma & \text { Specific heat ratio } \\ \eta & \text { Efficiency } \\ Y^{+} & \text {Non-dimensional wall distance }\end{array}$

\section{Subscripts}

$\begin{array}{ll}\text { Max } & \text { Maximum } \\ \text { Min } & \text { Minimum } \\ \text { Ave } & \text { Average } \\ \text { Peak } & \text { Adiabatic efficiency at peak } \\ \text { in } & \text { Inlet } \\ \text { out } & \text { Outlet } \\ \text { SW } & \text { Smooth Wall }\end{array}$

\section{References}

1. Schlechtriem, S. and Lötzerich, M. "Breakdown of tip leakage vortices in compressors at flow conditions close to stall", ASME Paper 97-GT-41 (1997).

2. März, J., Hah, C., and Neise, W. "An experimental and numerical investigation into the mechanisms of rotating instability", Journal of Turbomachinery, 124(3), pp. 367-374 (2001).

3. Juan, D., Jichao, L., Lipeng, G., Feng., et al. "The impact of casing groove location on stall margin and tip clearance flow in a low-speed axial compressor", Journal of Turbomachinery, 138(12), pp. 1-11 (2016).

4. Bailey, E.E. "Effect of grooved casing treatment on the flow range capability of a single-stage axial-flow compressor", NASA TM-X2459 (1972).

5. Heinichen, F., Gummer, V., and Schiffer, H.-P. "Numerical investigation of a single circumferential groove casing treatment on three different compressor rotors", Proceedings of the ASME Turbo Expo, GT2011-45905, pp. 201-211(2011).

6. Shi, K., Chen, H., and Fu, S. "Numerical investigation of the casing treatment mechanism with a single circumferential groove", Science China Physics, Mechanics and Astronomy, 56(2), pp. 353-365 (2013).

7. Li, J., Lin, F., Wang, S., et al. "Extensive experimental study of circumferential single groove in an axial flow compressor", Proceedings of ASME Turbo Expo, pp. $1-13$ (2014).

8. Yasunori, S., Toshinori, W., Takehiro, H., et al. "Numerical analysis of flow in a transonic compressor with a single circumferential casing groove: influence of groove location and depth on flow instability", Journal of Turbomachinery, $\mathbf{1 3 6}(3)$, pp. 1-9 (2014).

9. Wang, W., Lu-J, L., Luo, X.-Q., et al. "Coupling method of stability enhancement based on casing treatments in an axial compressor", Aerospace Science and Technology, 95, pp. 1-13 (2019).

10. Xi, N., Ma, N., Lin, F., et al. "A new approach of casing treatment design for high speed compressors running at partial speeds with low speed large scale test", Aerospace Science and Technology, 72, pp. 104113 (2018).

11. Dinh, C.-T., Heo, M.-W., and Kim, K.-Y. "Aerodynamic performance of transonic axial compressor with a casing groove combined with blade tip injection and ejection", Aerospace Science and Technology, 46, pp. 176-187 (2015).

12. Azizi, R., Ebrahimi, R., and Ziabasharhagh, M., "Algorithm development for aerodynamic preliminary design of multi-stage axial compressors", Scientia Iranica, 23(5), pp. 2166-2178 (2016). 
13. Zhang, H., Wenhao, L., Enhao, W., et al. "Mechanism investigation of enhancing the stability of an axial flow rotor by blade angle slots", Proceedings of the Institution of Mechanical Engineers, Part G: Journal of Aerospace Engineering, 233(13), pp. 4750-4764 (2019).

14. Le, L., Xi, N., and Lin, F. "Flow measurements near the open surfaces of single circumferential grooves in a low-speed axial compressor", Aerospace Science and Technology, 78, pp. 531-541 (2018).

15. Li, J., Juan, D., Yang, L., et al. "Effect of inlet radial distortion on aerodynamic stability in a multistage axial flow compressor", Aerospace Science and Technology, 105, pp. 1-11 (2020).

16. Madadi, A., Kermani, M., and Nili-Ahmadabadi, M. "Application of the ball-spine algorithm to design axial-flow compressor blade", Scientia Iranica, 21(6), pp. 1981-1992 (2014).

17. Wang, W., Wuli, C., Haoguang, Z., et al. "The effects on stability, performance, and tip leakage flow of recirculating casing treatment in a subsonic axial flow compressor", Proceedings of the ASME Turbo Expo, 2.A, pp. 1-12 (2016).

18. Kim, J.-H., Choi, K.-J., and Kim, K.-Y. "Aerodynamic analysis and optimization of a transonic axial compressor with casing grooves to improve operating stability", Aerospace Science and Technology, 29(1), pp. 81-91 (2013).

19. Yan, S. and Wuli, C. "The improvement of transonic compressor performance by the self-circulating casing treatment", Proceedings of the Institution of Mechanical Engineers, Part C: Journal of Mechanical Engineering Science, 235(7), pp. 1165-1176 (2020).

20. Kawase, M. and Aldo. R. "Numerical optimization of a stall margin enhancing recirculation channel for an axial compressor", Fluids, 4(2), pp. 1-19 (2019).

21. Zhao, Q., Zhou, X., and Xiang, X. "Multi-objective optimization of groove casing treatment in a transonic compressor", Proceedings of the Institution of Mechanical Engineers, Part A: Journal of Power and Energy, 228(6), pp. 626-637 (2014).

22. Li, J., Du, J., Li, F., et al. "Stability enhancement using a new hybrid casing treatment in an axial flow compressor", Aerospace Science and Technology, 85, pp. 305-319 (2019).

23. Mao, X. and Liu, B. "Investigation of the casing groove location effect for a large tip clearance in a counterrotating axial flow compressor", Aerospace Science and Technology, 105, pp. 1-12 (2020).

24. Naseem, A., Qun, Z., Hamza, F., et al. "Axial transonic compressor performance enhancement with circumferential grooves", Mechanical Sciences, 11(1), pp. 153-161 (2020).

25. Zhu, G. and Yang, B. "Optimization of slots-groove coupled casing treatment for an axial transonic compressor", Journal of Turbomachinery, 142(8), pp. 1-12 (2020).
26. Du, J., Li, J., Wang, K., et al. "The self-induced unsteadiness of tip leakage flow in an axial lowspeed compressor with single circumferential casing groove", Journal of Thermal Science, 22(6), pp. 565572 (2013).

27. Liu, L., Li, J., Nan, X., et al. "The stall inceptions in an axial compressor with single circumferential groove casing treatment at different axial locations", Aerospace Science and Technology, 59, pp. 145-154 (2016).

28. Naseem, A., Qun, Z., Hamza, F., et al. "Performance improvement of axial compressor by introduction of circumferential grooves", Energy Sources, Part A: Recovery, Utilization, and Environmental Effects, pp. $1-21$ (2020).

29. Ashok, A., Sidhartha, P.A.V., and Sivadasan, S. "Effect of various trench designs on axial compressor blade tip aerodynamics", ASME Gas Turbine India Conference, American Society of Mechanical Engineers Digital Collection, pp. 1-10 (2019).

30. Wang, W., Lu-J, L., Luo, X.-Q., et al. "Failure mechanism of casing treatment in improving stability of a highly loaded two-stage axial compressor", Aerospace Science and Technology, 105, pp. 1-13 (2020).

31. Hamzezade, M., Agha Seyed Mirzabozorg, M., and Bazazzadeh, M. "Numerical study of the effect of the tip gap size and using a single circumferential groove on the performance of a multistage compressor", Journal of Computational Applied Mechanics, 50(1), pp. 54-62 (2019).

32. Dunham, J. "CFD validation for propulsion system components", AGARD advisory Report no. 355, advisory group on aerospace research and development, North Atlantic treaty organization, ISBN 92-836-1075X. (1998).

33. Kim, J.H., Kim, K-Y., and Cha, K.H. "Effects of number of circumferential casing grooves on stall flow characteristics of a transonic axial compressor", Applied Mechanics and Materials, 284-287, pp. 727-732 (2013).

34. Huang, X., Chen, H., and Fu, S. "CFD investigation on the circumferential grooves casing treatment of transonic compressor", ASME Turbo Expo: Power for Land, Sea, and Air, 43161, pp. 581-589 (2008).

35. ANSYS CFX-17.0.0., ANSYS CFX-solver Theory Guide. Release, ANSYS Inc. (2016).

36. NUMECA International, IGG v8.9-1 and Autogrid5 v8.9-1, User Manual, Brussels: NUMECA International (2009).

37. Naseem, A., Bin, J., Qun, Z., et al. "Performance enhancement of a transonic axial flow compressor with circumferential casing grooves to improve the stall margin", Journal of Applied Fluid Mechanics, 13(1), pp. 221-32 (2019). 


\section{Biographies}

Naseem Ahmad is a Research Assistant at Harbin Engineering University and currently perusing a PhD degree in the field of Power Engineering and Engineering Thermophysics from the College of Power and Energy Engineering. He has eight years of strong industrial and academic experience in the field of Mechanical and Power Engineering. His research area includes computational fluid dynamics, turbine blade cooling, aerodynamics of turbomachinery, alternative fuels. He has published 11 SCI and 3 EI papers in various international reputed journals.

Qun Zheng is a Full Professor in the CPEE, HEU China. He received his BS and MS degrees in Marine Engineering from HEU and HIT in 1984 and 1987, respectively, and his $\mathrm{PhD}$ degree in 2000 from Harbin Engineering University, in the area of turbomachinery. His area of research includes aerodynamics, gas turbine and steam power plant, turbine blade cooling and thermodynamics, etc. He has published more than 350 SCI and EI research papers in various SCI and EI journals.

Hamza Fawzy is a Research Assistant in the College of Power and Energy Engineering, Harbin Engineering University. He has worked as assistant lecturer in Mechanical Power Engineering, Menofia University for
4 years. His research interests include aerodynamics of turbomachinery, and novel techniques of blade cooling of gas turbine blades. He has published 11 papers indexed by SCI.

Aqiang Lin has received his PhD degree in 2020 from the College of Power and Energy Engineering, Harbin Engineering University, China. Currently he is working as lecturer at School of Power and Energy, Northwestern Polytechnical University, Xi'an. His research interests include thermodynamics, aerodynamics, turbine blade cooling, gas turbine, and steam power plant. He has published more than 30 research publications in different SCI journals.

Bin Jiang is working as an Associate Professor in the College of Power and Energy Engineering, Harbin Engineering University, China. He graduated from Harbin Institute of Technology in 2010 with a $\mathrm{PhD}$ degree in power machinery and engineering. He received postdoctoral degree in Power Engineering and Engineering Thermophysics at Harbin Engineering University. His current research direction is the aero-thermodynamics of gas turbine compressors including the aerodynamic design methods of high-performance compressors, the mechanism of compressor instability and expansion methods, etc. He has published more than 30 SCI papers in different international journals. 